\title{
Mesures et calcul des doses délivrées par des particules radioactives
}

\author{
J.B. LEROUX*, Y. HERBAUT*
}

(Manuscrit reçu le 26 juin 1995, révisé le 12 mars 1996, accepté le 3 octobre 1996)

RÉSUMÉ Les accidents de Three Mile Island (TMI) aux USA et Tchernobyl en Ukraine ont mis en évidence les risques importants d'irradiation de la peau par de petites particules radioactives, appelées particules chaudes (hot particles, en anglais) constituées de fragments de combustibles ou de matériaux irradiés qui ont été activés dans le réacteur en passant près du cour. Ces risques d'irradiation sont dus essentiellement aux rayonnements $\beta$ émis par ces particules. Différents organismes ou groupes de travail ont examiné ces risques (AIEA, ICRP, NCRP) et ont déterminé des doses seuils qui devraient éviter l'ulcération de la peau. Dans le cadre d'un contrat de recherche de la Commission des communautés européennes, plusieurs laboratoires travaillent en collaboration pour valider des méthodes expérimentales et des codes de calcul permettant de déterminer les doses délivrées à la peau par ces particules chaudes. Ce document présente la comparaison des résultats obtenus au moyen d'une chambre à extrapolation et de deux codes de calcul (Code Varskin Mod 2 et Code Monte-Carlo HOT 25 S2) pour l'évaluation de la dose délivrée à proximité d'une source de ${ }^{60} \mathrm{Co}$ de $200 \mathrm{~mm}$ de diamètre. Les deux codes de calcul donnent des résultats comparables, mais environ deux fois plus faibles que ceux de la chambre à extrapolation. La mesure du débit de dose $[\beta+\gamma]$ sous $7 \mathrm{mg} \mathrm{cm}^{-2}$, délivré par une source de ${ }^{60} \mathrm{Co}$ de $200 \mu \mathrm{m}$ de diamètre, au contact de la peau, donne $6,11^{-2} \mathrm{nGy} \mathrm{s}^{-1} \mathrm{~Bq}^{-1}$ pour une surface irradiée égale à $1 \mathrm{~cm}^{2}$.

ABSTRACT Three Mile Island (TMI) and Chernobyl reactor accidents have revealed the importance of the skin exposure to beta radiation produced by small high activity sources, named "hot particles". In nuclear power reactors, they may arise as small fragments of irradiated fuel or material which have been neutron activated by passing through the reactor co. In recent years, skin exposure to hot particles has been subject to different limitation criteria, formulated by AIEA, ICRP, NCRP working groups. The present work is the contribution of CEA Grenoble to a contract of the Commission of the European communities in cooperation with several laboratories : University of Birmingham, University of Toulouse and University of Montpellier with the main goal to check experiments and calculations of tissue dose from ${ }^{60} \mathrm{Co}$ radioactive particles. This report is split up into two parts : 1) Hot particle dosimetry close to a ${ }^{60} \mathrm{Co}$ spherical sample with an approximately $200 \mu \mathrm{m}$ diameter, using a PTW extrapolation chamber model 23391. 2) Dose calculations from two codes : the Varskin Mod 2 computer code and the Hot 25 S2 MonteCarlo algorithm. The two codes lead to similar results; nevertheless there is a large discrepancy (of about 2) between calculations and PTW measurements which are higher by a factor of 1.9. At a $70 \mu \mathrm{m}$ skin depth and for $1 \mathrm{~cm}^{2}$ irradiated area, the total $(\beta+\gamma)$ tissue dose rate delivered by a spherical $(\phi=200 \mu \mathrm{m}){ }^{60} \mathrm{Co}$ source, in contact with skin, is of the order of $6.11^{-2} \mathrm{nGy} \mathrm{s}^{-1} \mathrm{~Bq}^{-1}$.

Service de Protection contre les Rayonnements; CEA/Grenoble, 17 rue des Martyrs, 38054 Grenoble, Cedex 9, France. 


\section{Introduction}

L'évaluation des doses délivrées à la peau par de très petites sources radioactives constituées d'émetteurs $\beta$ et $\gamma$ s'est posée de façon cruciale à la suite des accidents de Three Mile Island et de Tchernobyl. Différentes publications récentes mettent en évidence l'existence de telles particules dans les réacteurs (SNE, 1994; EDF, 1994). Ces petites particules radioactives (de diamètre $<1 \mathrm{~mm}$ ), appelées en anglais "hot particles" sont constituées, dans un réacteur nucléaire, par de petits fragments de combustible ou de matériaux irradiés (généralement de l'acier) qui ont été activés en passant près du cœur. Les principaux radionucléides irencontrés sont des produits de fission pour les fragments de combustibles, ou du ${ }^{60}$ Co pour les fragments d'acier ; en effet, l'acier renferme uñ pourcentàge élevé de cobalt stable qui peut être activé dans le cour par la réaction ${ }^{59} \mathrm{Co}(\mathrm{n}, \gamma){ }^{60} \mathrm{Co}$.

Le risque le plus important présenté par de telles particules réside dans le fait qu'elles peuvent se déposer sur la peau et créer une irradiation importante de celle-ci, en raison des radionucléides émetteurs $\beta$ qu'elles peuvent contenir. De plus, cette irradiation est généralement non homogène, de très petites quantités de tissus pouvant être exposées à des doses très élevées.

Ces particules ne peuvent pas être détectées à l'œil, car leurs dimensions se situent généralement entre approximativement $1 \mu \mathrm{m}$ et quelques centaines de $\mu \mathrm{m}$. Elles sont apparemment chargées électriquement et, de ce fait, tendent à être très mobiles, “sautant” d'une surface à l'autre (NCRP, 1989).

L'activité des particules contenant des produits de fission est située entre $40 \mathrm{~Bq}$ et $400 \mathrm{kBq}(1 \mathrm{nCi}$ à $10 \mu \mathrm{Ci})$, la majorité étant comprise entre $400 \mathrm{~Bq}$ et $40 \mathrm{kBq}(10 \mathrm{nCi}$ à $1 \mu \mathrm{Ci})$. La radioactivité des particules de ${ }^{60} \mathrm{Co}$ se situe entre $40 \mathrm{~Bq}$ et $20 \mathrm{MBq}(1 \mathrm{nCi}$ à $500 \mu \mathrm{Ci})$, la majorité étant comprise entre $400 \mathrm{~Bq}$ et $200 \mathrm{kBq}(10 \mathrm{nCi}$ à $5 \mu \mathrm{Ci})$ (Warnock et al., 1987).

Récemment, différents groupes de travail (AIEA, ICRP, NCRP) ont examiné les effets biologiques des "hot particles" et ont recommandé une valeur limite, relative au produit de leur taux d'émission de particules $\beta$ et de la durée d'exposition (NCRP, 1989 ; ICRP, 1991). Ils définissent arbitrairement comme "hot particle" un petit fragment radioactif, insoluble dans l'eau, dont aucune des dimensions ne dépasse $1 \mathrm{~mm}$.

L'ICRP a suggéré une dose seuil de $1 \mathrm{~Sv}$ sur une surface $\cdot \mathrm{de} 1 \cdot \mathrm{cm}^{2}$ à une profondeur de 100 à $150 \mu \mathrm{m}\left(10\right.$ à $\left.15 \mathrm{mg} \mathrm{cm} \mathrm{cm}^{-2}\right)$, qui devrait éviter l'apparition d'ulcération transitoire de la peau (ICRP, 1991). Le NCRP a suggéré une limite d'exposition qui est donnée en nombre de particules bêta émises à la surface de la particule radioactive ; cette valeur limite devrait être égale à $10^{10}$ particules bêta.

De nombreux travaux expérimentaux ont été réalisés dans ce domaine, tant du point de vue des effets biologiques (Baum and Kaurin, 1991 ; Charles, 1991) 
que de la dosimétrie (Darley et al., 1991 ; Williams et al., 1992). Différents codes de calcul, ayant pour objectif l'évaluation de la dose délivrée par de telles particules (Durham et al., 1991a ; Durham, 1991b ; Durham, 1992 ; Chibani et Patau, 1994 ; Patau, 1991) ont été développés.

Dans le cadre d'un contrat de recherche des Communautés Européennes (Bi 7-021) une étude est menée par le Service de Protection contre les Rayonnements (SPR/SMI/LDI) du CEA de Grenoble, en collaboration avec l'Université de Birmingham (M.W. Charles, coordinateur du projet) l'Université de Toulouse III (J.P. Patau) et le Centre d'Électronique de Montpellier (J. Gasiot).

Cette étude a pour objectif principal la validation de codes de calcul des doses délivrées par des particules radioactives (hot particles) . de ${ }^{60} \mathrm{Co}$ et de ${ }^{170} \mathrm{Tm}$, par comparaison avec les résultats obtenus au moyen de différentes méthodes de mesure (chambre à extrapolation, dosimétrie par thermoluminescence, films radiochromiques).

Ce document présente les travaux réalisés par SPR/SMI/LDI concernant :

- la dosimétrie expérimentale à proximité d'une source radioactive de ${ }^{60} \mathrm{Co}$ de diamètre voisin de $200 \mu \mathrm{m}$, réalisée au moyen d'une chambre à extrapolation (Leroux et Herbaut, 1994).

- le calcul du débit de dose délivré par cette source au moyen de différents codes de calcul : le programme, par intégration numérique, Varskin Mod 2 de Durham (Durham, 1991a et b, 1992) et le code Monte-Carlo de Patau (Chibani et Patau, 1994 ; Patau, 1991).

Ultérieurement, nos résultats expérimentaux seront comparés à ceux obtenus par Charles au moyen de diverses techniques de mesure.

\section{Caractéristiques des moyens expérimentaux}

\subsection{Source radioactive}

La source utilisée est une source sphérique de ${ }^{60} \mathrm{Co}$ de $193 \mu \mathrm{m}$ de diamètre, fournie par Charles (référencée VIa), et réalisée par activation neutronique. La sphère de cobalt est montée au centre d'une pièce support circulaire, en aluminium (diamètre $13 \mathrm{~mm}$ ); elle est recouverte d'une feuille d'aluminium de $15 \mu \mathrm{m}$ ( $4 \mathrm{mg} \mathrm{cm}^{-2}$ ), et maintenue par de la colle (Fig. 1). Les caractéristiques principales de la source sont données dans le tableau I.

\subsection{Irradiateur}

Pour les différentes distances $d$ séparant la source et la fenêtre d'entrée de la chambre, la source de ${ }^{60} \mathrm{Co}$ est positionnée dans un irradiateur en plexiglas de $25 \mathrm{~mm}$ de long et $18 \mathrm{~mm}$ d'épaisseur. Un cylindre en acier est utilisé comme protection biologique et comme dispositif d'alignement. 


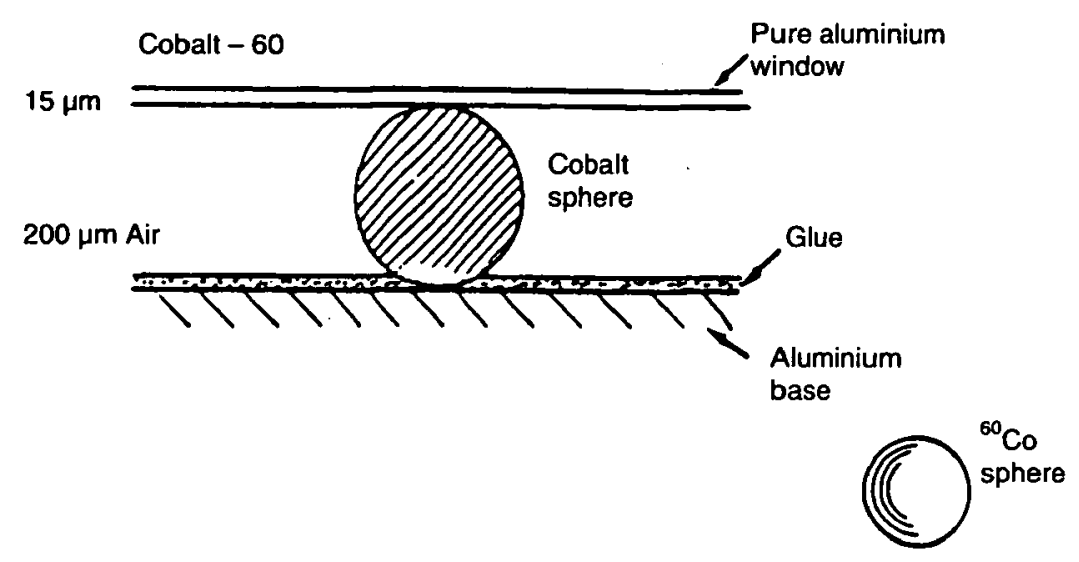

Fig. 1. - Schéma de la source de ${ }^{60} \mathrm{Co}$.

Schematic drawing of the ${ }^{60}$ Co source.

TABLEAU I

Caractéristiques de la source radioactive

Radioactive source properties

\begin{tabular}{|c|c|}
\hline Composition massique & 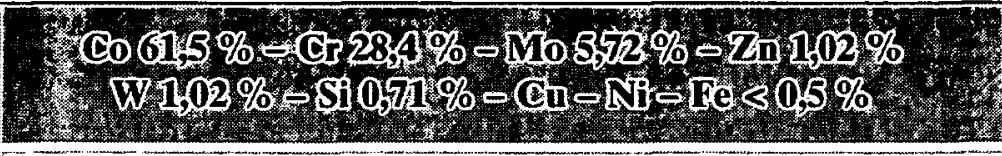 \\
\hline Masse & $3,010^{-5} \mathrm{~g}$ \\
\hline Masse volumique & $7,97 \mathrm{~g} \mathrm{~cm}^{-3}$ \\
\hline Radionucléide & ${ }^{60} \mathrm{Co}$ \\
\hline Période & $(5,271 \pm 0,002)$ ans \\
\hline Emission bêta & $\begin{array}{rlrl}\beta_{1}-E_{\max } & =317,9 \mathrm{keV} & 99,92 \% \\
E_{\operatorname{moy}} & =95,8 \mathrm{keV} & \\
\beta_{2}-E_{\max } & =1491,1 \mathrm{keV} & 0,08 \% \\
E_{\operatorname{moy}} & =627,4 \mathrm{keV} & \end{array}$ \\
\hline Emission gamma & $\begin{array}{ll}E_{\gamma 1}=1173,24 \mathrm{keV} & 99,89 \% \\
E_{\gamma 2}=1332,50 \mathrm{keV} & 99,98 \%\end{array}$ \\
\hline Activité & $\begin{array}{l}0,70 \mathrm{MBq}( \pm 3,0 \%) \text { le } 1.01 .1992(18,9 \mu \mathrm{Ci}) \text { mesurée } \\
\text { par spectrométrie } \gamma\end{array}$ \\
\hline
\end{tabular}




\subsection{Chambre à extrapolation}

\subsubsection{Généralités}

En dosimétrie, la chambre à extrapolation est considérée comme le détecteur le mieux adapté à la détermination de la dose absorbée délivrée par les rayonnements peu pénétrants, en particulier par les rayonnements $\beta$. Elle est considérée, pour ces rayonnements, comme le détecteur de référence par de nombreux laboratoires d'étalonnage (Böhm, 1986).

Si ce détecteur est réalisé en matériau équivalent au tissu et si il est équipé d'une fenêtre d'entrée d'épaisseur massique égale à $7 \mathrm{mg} \mathrm{cm}^{-2}$, il permet de déterminer le débit de dose absorbée dans le tissu à la profondeur de $7 \mathrm{mg} \mathrm{cm}^{-2}$.

Le principe de fonctionnement d'un tel détecteur et la méthodologie de mesure sont décrits en détails par Böhm (1986).

\subsubsection{Caractéristiques de la chambre à extrapolation}

La chambre à extrapolation de modèle 23391 utilisée ici est réalisée par la firme allemande PTW (Freiburg). Différentes fenêtres d'entrée en kapton graphité (film polyimide, de couleur ambrée, transparent, de masse volumique voisine de celle du mylar), d'épaisseur massique $4,5,8,5$ ou $12,5 \mathrm{mg} \mathrm{cm}^{-2}$, peuvent équiper la chambre. L'électrode collectrice, réalisée en matériau équivalent au tissu type A 150, est mobile. Son déplacement permet de faire varier l'épaisseur de la cavité de la chambre d'ionisation entre $0,6 \mathrm{~mm}$ et $25 \mathrm{~mm}$ (Fig. 2). Différentes électrodes collectrices, de diamètres : 10, 15, 20, 30, $40 \mathrm{~mm}$, peuvent équiper la chambre.

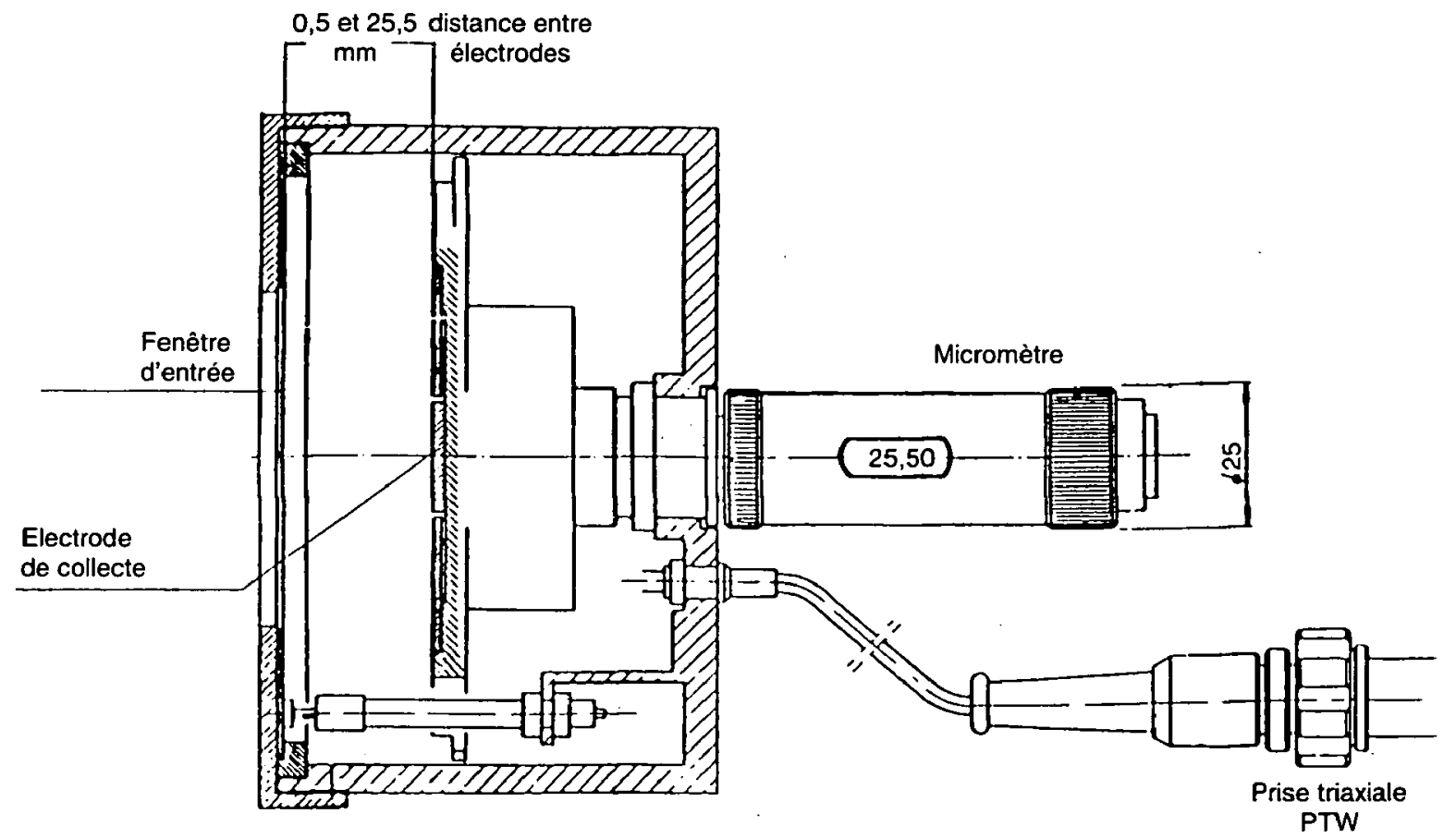

Fig. 2. - Chambre à extrapolation PTW modèle 23391.

Extrapolation chamber PTW model 23391. 
Pour les expériences décrites dans ce document, la chambre est équipée d'une fenêtre d'épaisseur massique égale à $12,5 \mathrm{mg} \mathrm{cm}^{-2}$ et d'une électrode collectrice de diamètre $10 \mathrm{~mm}$; le diamètre réel, déterminé expérimentalement, est égal à $10,7 \dot{\mathrm{mm}}( \pm 1 \%)$, ce qui correspond à une surface collectrice de $90 \mathrm{~mm}^{2}$. Le champ électrique appliqué à la chambre d'ionisation est constant et égal à $50 \mathrm{~V} \mathrm{~mm}^{-1}$.

La mesure du courant d'ionisation est réalisée par intégration au moyen d'un condensateur étalonné $(C=45,8 \mathrm{pF} \pm 0,01 \%)$, monté en contre - réaction sur un électromètre Keitlhey modèle 642.

Un calculateur H P Vectra ES 12 assure l'acquisition des informations (mesure de tension, pression, température, hygrométrie), leur traitement ainsi que le calcul des incertitudes.

\section{Caractéristiques des codes de calcul}

\subsection{Code Varskin Mod 2}

Le code de calcul Varskin Mod 2 écrit par Durham (Batelle Pacific Northwest Laboratory ; Durham, 1991a et b, 1992) permet de calculer la composante $\beta$ de la dose délivrée par une source radioactive en intégrant numériquement les distributions de dose autour de points source (Berger, 1973). Ces distributions, pour des énergies d'électrons aussi basses que $0,5 \mathrm{keV}$, prennent en compte la diffusion et le rayonnement de freinage et utilisent différents modèles suivant l'énergie ; l'intégration est réalisée sur toute la géométrie de la source, éventuellement en 3 dimensions. Les paramètres suivants peuvent être modifiés : géométrie de la source (disque, sphère, plaque), radionucléide(s) concerné(s) et activité(s), masse volumique de la source, caractéristiques physiques des écrans de protection (vêtements), distance d'air (en $\mathrm{mm}$ ) séparant la source et la peau, profondeur $\left(\mathrm{en} \mathrm{mg} \mathrm{cm}^{-2}\right)$ de tissu à laquelle le débit de dose doit être calculé.

Dans le cas des "hot particles"; pour un radionucléide émetteur $\beta$ et $\gamma$, la composante $\gamma$ peut être déterminée en utilisant le modèle simplifié de Lantz et Lambert (1990); dans ce cas, la source est supposée ponctuelle et la surface irradiée est égale à $1 \mathrm{~cm}^{2}$.

\subsection{Code Monte-Carlo "HOT $25 \mathrm{~S} 2$ "}

Le code de calcul Monte-Carlo "HOT $25 \mathrm{~S} 2$ " pour le transport des électrons a été écrit par Patau (Université de Toulouse III). Il ne calcule que la dose due au rayonnement $\beta$ (Chibani et Patau, 1994 ; Patau, 1991). Les électrons émis par une source sphérique homogène de ${ }^{60} \mathrm{Co}$, placée dans une configuration géométrique identique à celle de l'expérience, sont suivis jusqu'à une énergie de $30 \mathrm{keV}$; les dépôts d'énergie correspondants sont calculés pour une 
profondeur de tissu variable, dans un cylindre équivalent au tissu simulant la chambre à extrapolation employée (en particulier la fenêtre d'entrée).

Les paramètres suivants peuvent être modifiés : rayon de la source émettrice, densité de la source, distance d'air séparant source et cylindre, nombre de tranches dans le cylindre pour le calcul des dépôts d'énergie et de la variation de la dose en profondeur, nombre d'électrons primaires émis par la source.

Le microcalculateur utilisé (microprocesseur : 80486, fréquence : $100 \mathrm{MHz}$ ) effectue le transport de 100000 électrons en 10 minutes environ.

\section{Résultats expérimentaux}

\subsection{Mesures au moyen de la chambre à extrapolation PTW 23391}

\subsubsection{Détermination des termes correctifs de la chambre}

La détermination de la dose absorbée au moyen d'une chambre à extrapolation nécessite la détermination de nombreux termes correctifs, spécifiques du détecteur lui-même et des caractéristiques de l'expérience. Tous ces termes correctifs ont été déterminés pour les besoins de cette expérience, conformément à la méthodologie décrite par. Böhm (1986). Dans ce document, ne sera détaillée que la détermination du coefficient de divergence du faisceau $K_{\mathrm{di}}$ et du coefficient de transmission dans la fenêtre d'entrée de la chambre $K_{\mathrm{EW}}$.

- Coefficient de divergence du faisceau $K_{d i}$

La source, située à très petite distance de la chambre à extrapolation, irradie celle-ci de façon non homogène, créant ainsi une dose non homogène dans le volume collecteur. Le coefficient de correction de divergence du faisceau, $K_{\mathrm{di}}$, qui dépend de la distance source - chambre $d$, de la distance interélectrodes de la chambre $L$ et du rayon de l'électrode collectrice $a$, permet de corriger cette non homogénéité (Fig. 3) et est calculé par la relation (1) (Böhm, 1986).

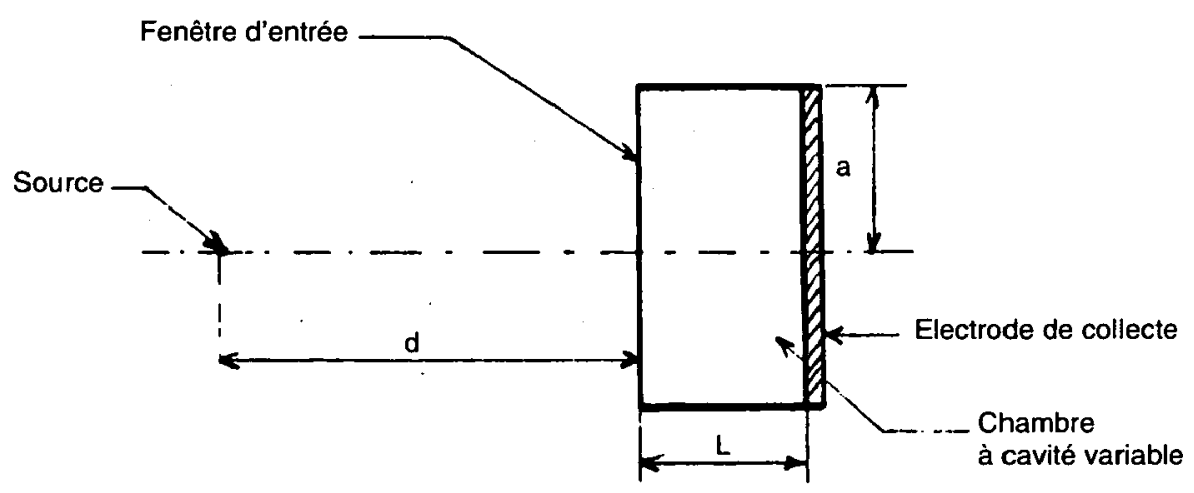

Fig. 3. - Facteur de correction $K_{d i}$ tenant compte de la divergence du faisceau. Correction factor $K_{d i}$ for the divergence of the beam. 


$$
K_{\mathrm{di}}=\frac{\dot{D}_{\mathrm{d}}}{\dot{D}}
$$

$\dot{D}_{\mathrm{d}}=$ débit de dose au niveau de la fenêtre d'entrée.

$\dot{D}$ = débit de dose moyen dans le volume collecteur de la chambre.

En réalisant l'intégration sur le volume de la chambre entre $d$ et $(d+L)$, $K_{\text {di }}$ est calculé par la relation (2).

$$
K_{\mathrm{di}}=\frac{\left[1-\left(\frac{d}{a}\right) \times \frac{1}{\sqrt{1+\left(\frac{d^{2}}{a^{2}}\right)}}\right] \times \frac{L}{a}}{\frac{L}{a}-\sqrt{1+\frac{(d+L)^{2}}{a^{2}}}+\sqrt{1+\frac{d^{2}}{a^{2}}}}
$$

Pour une électrode collectrice de $10,7 \mathrm{~mm}$ de diamètre, une distance interélectrodes de $1 \mathrm{~mm}$ et une distance source - chambre de $1 \mathrm{~mm}$ :

$$
K_{\mathrm{di}}=1,15
$$

Dans le cas d'une source de cobalt 60 , la dose absorbée mesurée par la chambre possède deux composantes : une composante bêta de faible énergie et une composante gamma de haute énergie. Le coefficient de correction $K_{\mathrm{di}}$ est appliqué aux deux composantes.

- Coefficient de correction relatif à l'épaisseur de la fenêtre d'entrée de la chambre à extrapolation $K_{E W}$

Pour le rayonnement $\beta$, la grandeur de référence est le débit de dose dans le tissu sous $7 \mathrm{mg} \mathrm{cm}{ }^{-2}$ de tissu, $D_{t, 7}^{0}(\beta)$, dans un fantôme semi-infini de tissu.

La chambre à extrapolation étant équipée pour ces mesures d'une fenêtre d'entrée en kapton d'épaisseur massique $12,5 \mathrm{mg} \mathrm{cm}^{-2}$ (épaisseur équivalente à $11,5 \mathrm{mg} \mathrm{cm}^{-2}$ de tissu), il est nécessaire d'introduire un terme correctif, $K_{\mathrm{EW}}$, pour tenir compte de la différence de transmission entre 11,5 et $7 \mathrm{mg} \mathrm{cm}$.

Soit $\dot{D}_{\mathrm{t}, x_{\mathrm{t}}}$ le débit de dose dans le tissu sous l'épaisseur équivalente de tissu $x_{\mathrm{t}}$ correspondant à l'épaisseur $x_{\mathrm{k}}$ de kapton de la fenêtre d'entrée de la chambre.

$\dot{D}_{\mathrm{t}, x_{\mathrm{t}}}$ est déterminé à partir des mesures de débit de dose dans l'air de la cavité dé la chambre $\dot{D}_{\mathrm{a}}, x_{\mathrm{t}}$, équipée d'une fenêtre d'entrée $x_{\mathrm{t}}$, en appliquant la théorie de Bragg-Gray: 
MESURE ET CALCUL DES DOSES DÉLIVRÉES PAR DES PARTICULES RADIOACTIVES

$$
\dot{D}_{\mathrm{t}, x_{\mathrm{t}}}=S_{\mathrm{t}, \mathrm{a}} \times \dot{D}_{\mathrm{a}, x_{\mathrm{t}}}
$$

$S_{\mathrm{t}, \text { a }}$ est le rapport des pouvoirs d'arrêt tissu-air.

Pour le spectre $\beta$ du ${ }^{60} \mathrm{Co}, S_{\mathrm{t}, \text { a }}$ est calculé à partir des données de Cross $e t$ al. (1989) et ICRU (1984).

$$
S_{\mathrm{t}, \mathrm{a}}=1,129
$$

Pour le rayonnement $\gamma, S_{\mathrm{t}}$ a est remplacé dans la relation (3) par le rapport des coefficients massiques d'absorption en énergie du tissu et de l'air $\left[\mu_{\mathrm{en}}\right]_{\mathrm{t}, \mathrm{a}}$.

$$
\left[\mu_{e n}\right]_{t, a}=1,102
$$

Pour une distance source-chambre égale à $0,2 \mathrm{~mm}, \dot{D}_{\mathrm{t}, x_{1}}$ est mesuré en équipant successivement la chambre des fenêtres d'épaisseur de kapton $x_{\mathrm{k}}: 4,5$ $-8,5-12,5 \mathrm{mg} \mathrm{cm}^{-2}$. A partir des données de Cross (1986) et en assimilant le kapton au mylar, le facteur d'atténuation relative (fonction du numéro atomique des milieux et égal dans ce cas à 0,92 ) permet de convertir les épaisseurs de kapton en épaisseurs de tissu.

$$
x_{\mathrm{t}}=0,92 x_{\mathrm{k}}
$$

Les résultats sont présentés sur la figure 4. A partir de ces données, par interpolation, on détermine la valeur de $K_{\mathrm{EW}}$ :

$$
\begin{aligned}
& K_{\mathrm{EW}}=\frac{\dot{D}_{\mathrm{t}, 7}}{\dot{D}_{\mathrm{t}, 11,5}} \\
& K_{\mathrm{EW}}=1,63
\end{aligned}
$$

Nous supposerons que ce coefficient est indépendant de la distance sourcechambre. Il n'est pris en compte que pour la composante $\beta$, l'influence de l'épaisseur de la fenêtre d'entrée étant négligeable pour la composante $\gamma$.

\subsubsection{Détermination du débit de dose absorbée}

Pour toutes les distances source-chambre réalisées, les débits de dose $\beta$ et $\gamma$ dans le tissu sous $7 \mathrm{mg} \mathrm{cm}^{-2}, \dot{D}_{\mathrm{t}, 7}(\beta)$ et $\dot{D}_{\mathrm{t}, 7}(\gamma)$, sont calculés à partir des relations (6) et (7).

$$
\begin{aligned}
& \dot{D}_{\mathrm{t}, 7}(\beta)=\frac{2,810^{5} \times K_{\mathrm{EW}} \times \dot{D}_{\mathrm{t}, 11,5}(\beta)}{A} \\
& \dot{D}_{\mathrm{t}, 11,5}(\beta)=S_{\mathrm{t}, \mathrm{a}} \dot{D}_{\mathrm{a}, 11,5}(\beta)
\end{aligned}
$$




$$
\begin{aligned}
& \dot{D}_{\mathrm{t}, 7}(\gamma)=\frac{2,810^{5} \times \dot{D}_{\mathrm{t}, 11,5}(\gamma)}{A} \\
& \dot{D}_{\mathrm{t}, 11,5}(\gamma)=\left[\mu_{\mathrm{en}}\right]_{\mathrm{t}, \mathrm{a}} \dot{D}_{\mathrm{a}, 11,5}(\gamma)
\end{aligned}
$$

$\dot{D}_{\mathrm{t}, 7}(\beta)$ et $\dot{D}_{\mathrm{t}, 7}(\gamma)$ sont exprimés en nGy s${ }^{-1} \mathrm{~Bq}^{-1}$.

$\dot{D}_{\mathrm{t}, 11,5}(\beta)$ et $\dot{D}_{\mathrm{t}, 11,5}(\gamma)$ sont les débits de dose $\beta$ et $\gamma$ dans le tissu sous $11,5 \mathrm{mg} \mathrm{cm}^{-2}$ de tissu $\left(12,5 \mathrm{mg} \mathrm{cm}^{-2}\right.$ de kapton) à la date de référence, exprimés en $\mathrm{Gy} \mathrm{h}^{-1}$.

$A$ est l'activité de la source (Bq) à la date de référence (le 01.01.1992).

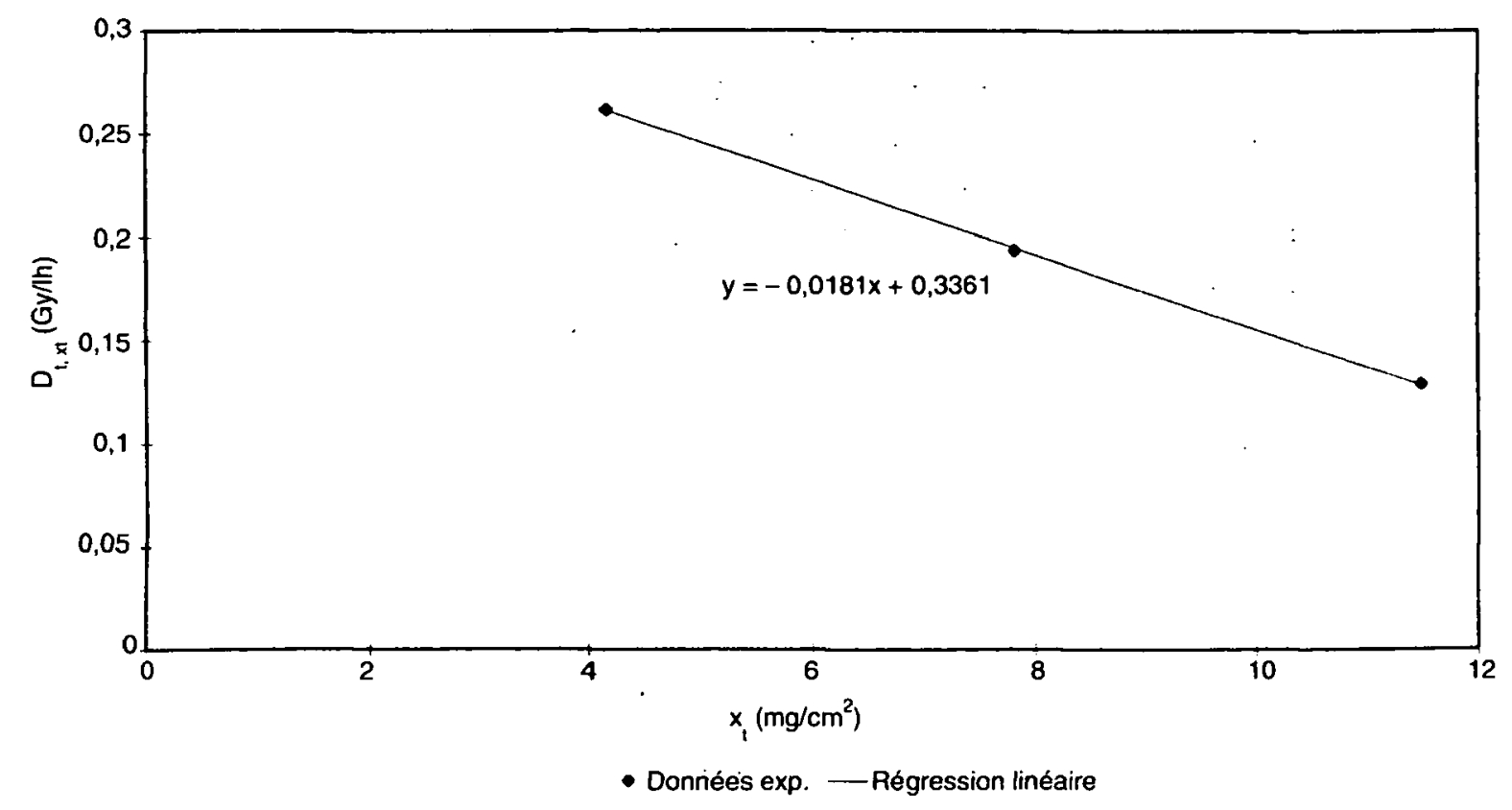

Fig. 4. - Influence de l'épaisseur de la fenêtre d'entrée.

Entrance window thickness influence.

\subsubsection{Détermination de la composante gamma}

La composante $\gamma$ est déterminée en interposant un écran entre source et détecteur pour arrêter complètement la composante $\beta$. Pour $d=2,3 \mathrm{~mm}$, l'écran est constitué par un disque en plexiglas (épaisseur $1,13 \mathrm{~mm}$ ); pour $d=1 \mathrm{~mm}$, il est constitué par un disque en cuivre (épaisseur $0,2 \mathrm{~mm}$ ). Pour $d=0,2$ et $0,5 \mathrm{~mm}$, il n'est pas possible d'interposer un écran ; la composante $\gamma$ est considérée comme égale à celle mesurée pour $d=1 \mathrm{~mm}$.

\subsubsection{Résultats}

La figure 5 présente une droite d'extrapolation " $\beta+\gamma$ " pour $d=1 \mathrm{~mm}$ et une distance interélectrodes comprise entre 0,7 et $1,2 \mathrm{~mm}$. Les valeurs de $\dot{D}_{\mathrm{t}, 7}(\beta)$ et $\dot{D}_{\mathrm{t}, 7}(\gamma)$ sont présentées dans le tableau II. 


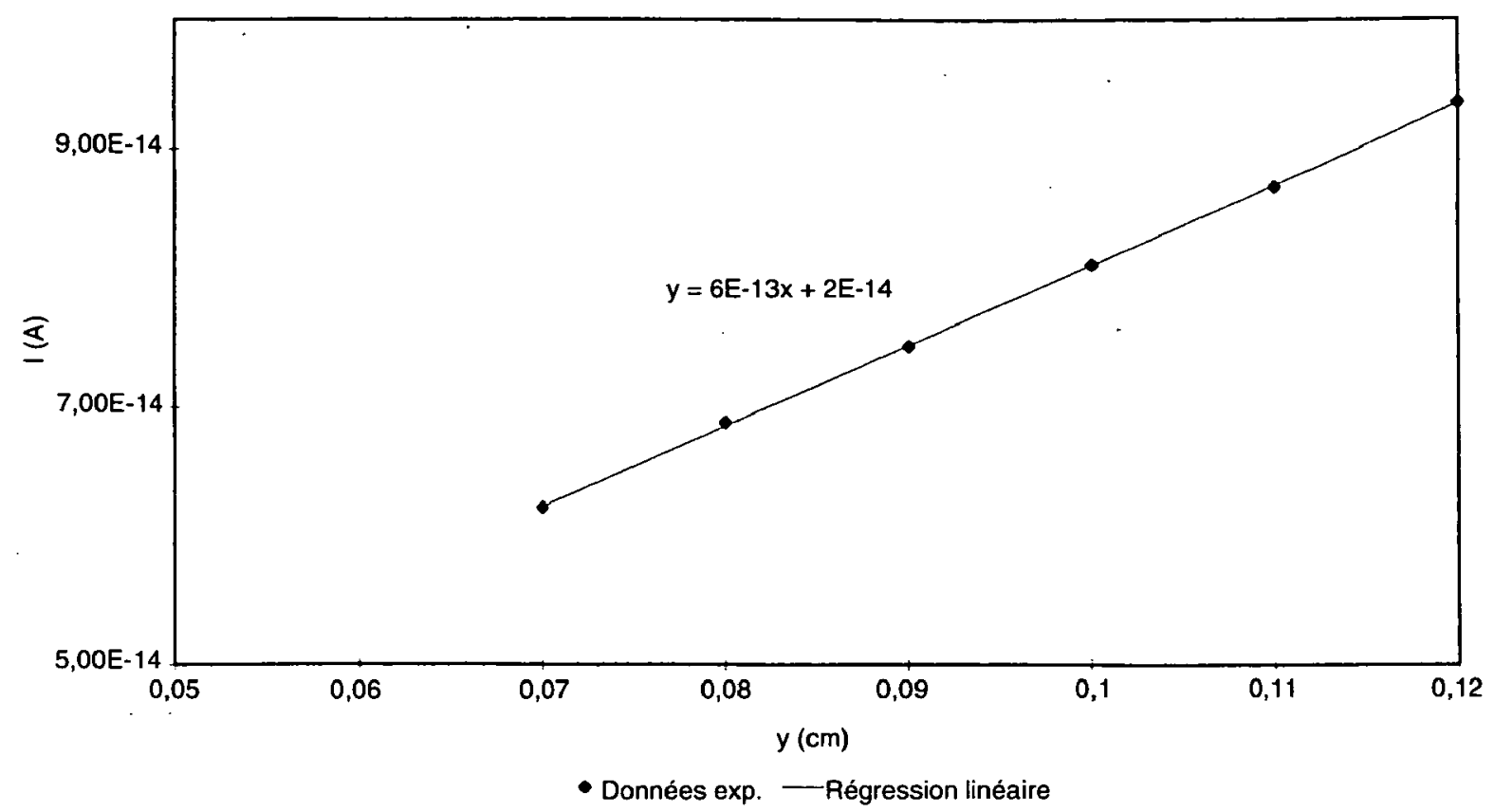

Fig. 5. - Droite d'extrapolation " $\beta+\gamma$ ".

Extrapolation curve for the whole radiation.

TABLEAU II

Débits de dose absorbée $\beta$ et $\gamma$ mesurés au moyen de la chambre à extrapolation $\beta$ and $\gamma$ absorbed dose rates carried out with the extrapolation chamber

\begin{tabular}{|c|c|c|c|}
\hline $\begin{array}{r}\text { Distanc } \\
\text { source-cha } \\
\text { (mim) } \\
\end{array}$ & $2+8 x^{2}$ & imbre a extrapola & 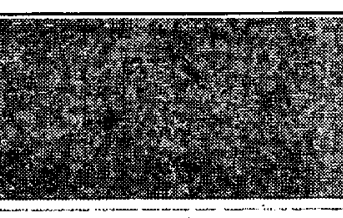 \\
\hline & $\begin{array}{c}\dot{D}_{\mathrm{t}, 7}(\beta) \\
\left(\mathrm{nGy} \mathrm{s}^{-1} \mathrm{~Bq}^{-1}\right)\end{array}$ & $\begin{array}{c}\dot{D}_{\mathrm{t}, 7}(\gamma) \\
\left(\mathrm{nGy} \mathrm{s}^{-1} \mathrm{~Bq}^{-1}\right)\end{array}$ & $\begin{array}{l}\text { Ecart-type relatif } \\
\text { sur } \dot{D}_{\mathrm{t}, 7}(\beta)\end{array}$ \\
\hline 0,2 & $5,5910^{-2}$ & $5,0110^{-3 *}$ & $29 \%$ \\
\hline 0,5 & $5,1810^{-2}$ & $5,0110^{-3 *}$ & $23 \%$ \\
\hline 1 & $5,0410^{-2}$ & $5,0110^{-3}$ & $12 \%$ \\
\hline 2 & $3,9510^{-2}$ & $4,4010^{-3}$ & $6 \%$ \\
\hline 3 & $3,0910^{-2}$ & $3,5410^{-3}$ & $4 \%$ \\
\hline
\end{tabular}

(1) La composante $\gamma$ est supposée indépendante de l'épaisseur de la fenêtre d'entrée.

(2) Les valeurs notées * ne sont pas des valeurs mesurées.

Les valeurs pour $d=0,2$ et $0,5 \mathrm{~mm}$ sont supposées égales à la valeur pour $d=1 \mathrm{~mm}$. 
Les variations de $\dot{D}_{\mathbf{t}, 7}(\beta)$ et $\dot{D}_{\mathrm{t}, 7}(\beta+\gamma)$ en fonction de $d$ sont représentées respectivement en figures 6 et 7 .

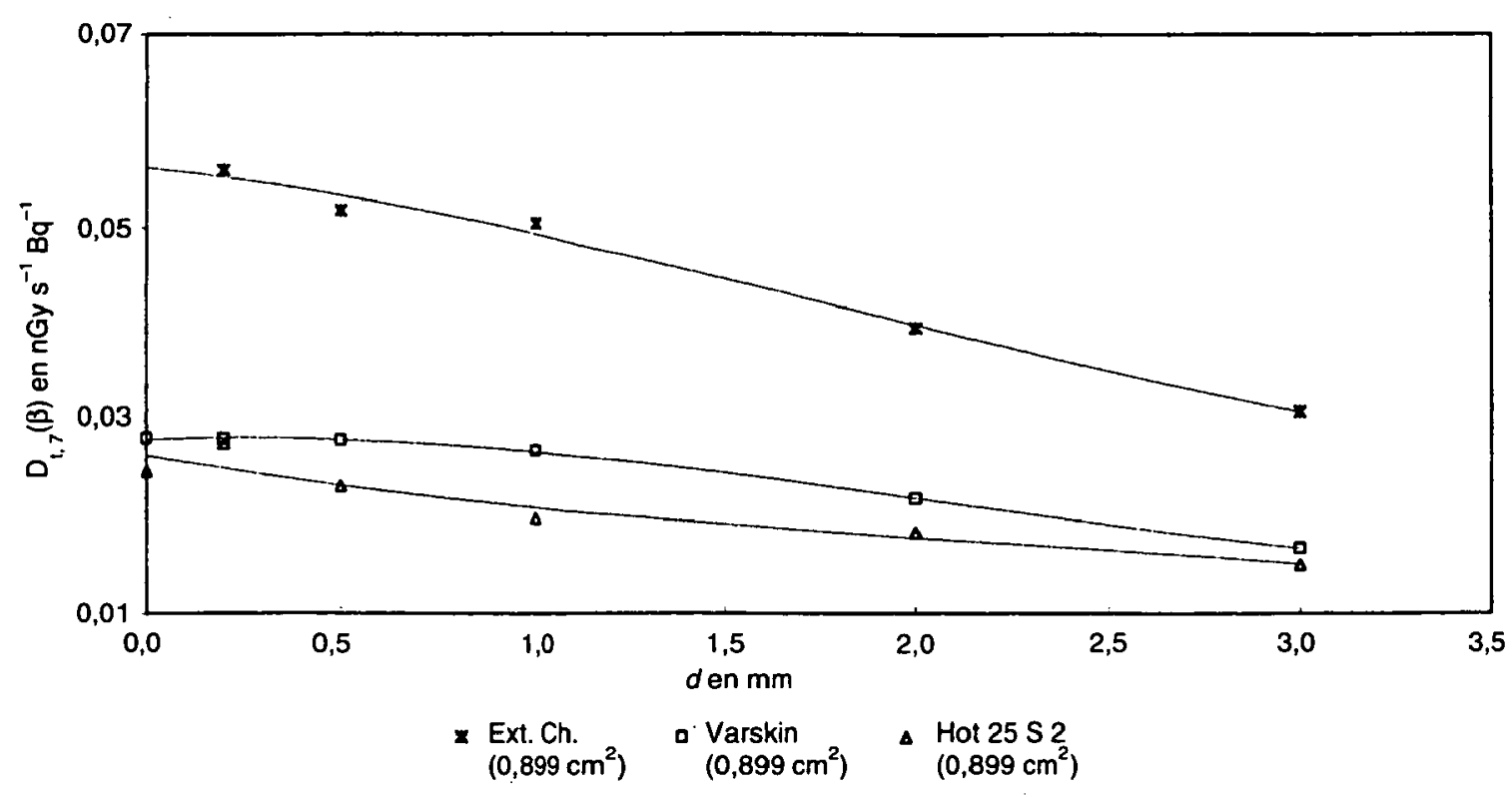

Fig. 6. - Variation du débit de dose bêta dans le tissu avec la distance source-peau. Beta tissue dose rate variation with source-skin distance.

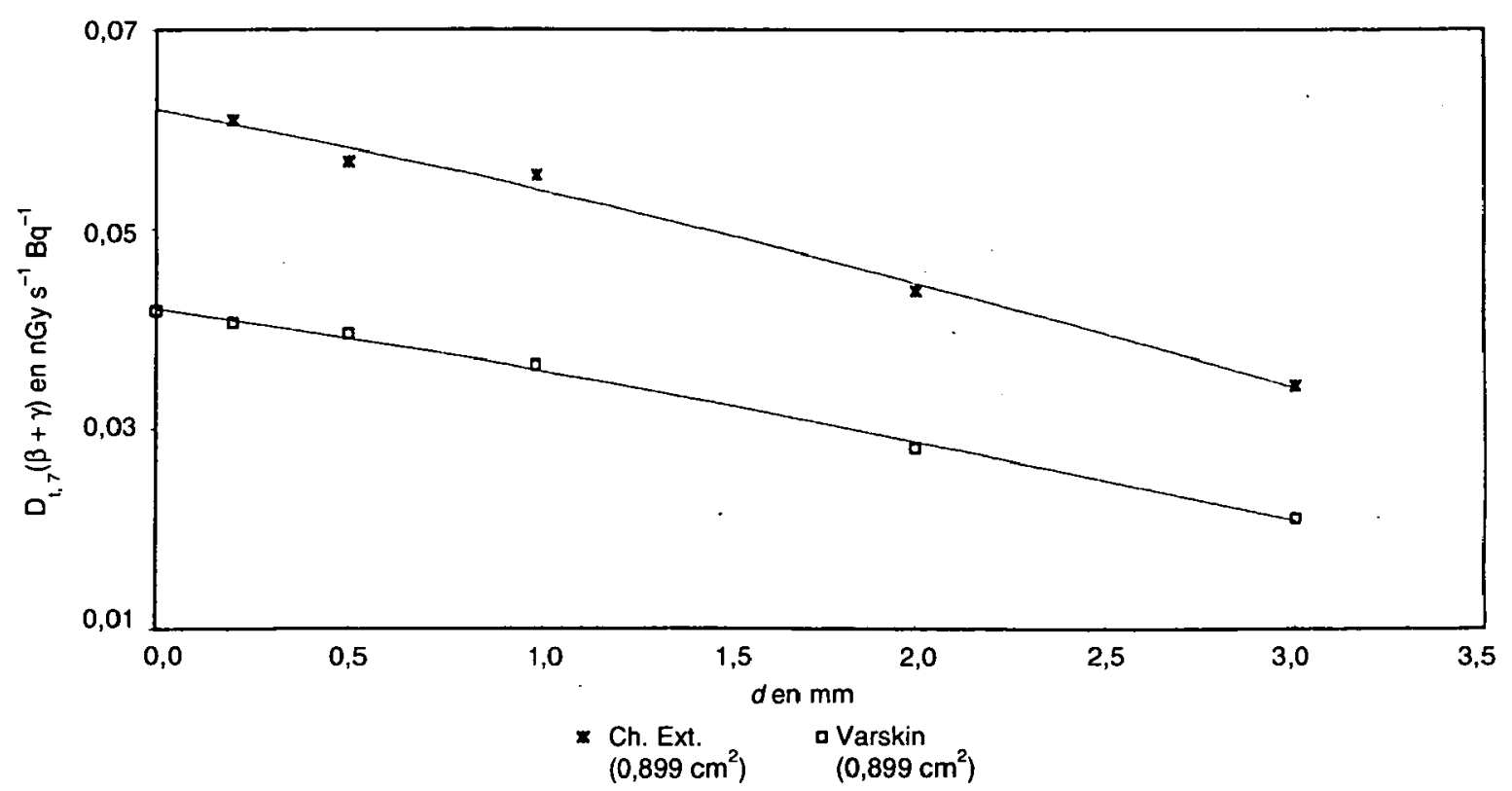

Fig. 7. - Variation du débit de dose total dans le tissu avec la distance source-peau. Total tissue dose rate variation with source-skin distance. 


\subsection{Détermination du débit de dose au moyen de codes de calcul et comparaison avec les résultats expérimentaux}

\section{a) Code Varskin Mod 2}

Les calculs avec le Code Varskin Mod 2 sont effectués pour une surface égale à celle de l'électrode de la chambre, soit $0,899 \mathrm{~cm} 2$, et une erreur relative de 0,001 . Les résultats obtenus sont comparés aux valeurs expérimentales, dans le tableau III et la figure 6 pour $\dot{D}_{\mathrm{t}, 7}(\beta)$ et dans le tableau IV et la figure 7 pour $\dot{D}_{\mathrm{t}, 7}(\beta+\gamma)$.

Les débits de dose absorbée dus au rayonnement $\beta$ mesurés par la chambre à extrapolation PTW sont environ 1,9 fois plus élevés que les résultats donnés par le code de calcul Varskin : l'écart devient voisin de 1,5 fois lorsque l'on prend en compte la composante gamma.

Ces conclusions sont semblables à celles obtenues par Charles dans le rapport CEGB "CEC hot particle dosimetry project" (Charles et Darley, 1994), dans lequel sont comparés, pour la même source de ${ }^{60} \mathrm{Co}$, les résultats donnés par une chambre à extrapolation de construction différente et les valeurs obtenues par le Code Varskin Mod 2.

Les résultats de calcul obtenus pour $d=0$ (au contact), 0,2 $\mathrm{mm}$ et $1 \mathrm{~mm}$, pour la profondeur de $7 \mathrm{mg} \mathrm{cm}-2$ et pour une surface irradiée variant de

\section{TABLEAU III}

Comparaison des résultats expérimentaux et des codes de calculs pour la composante $\beta$

Comparison for the beta component between experiments and computations

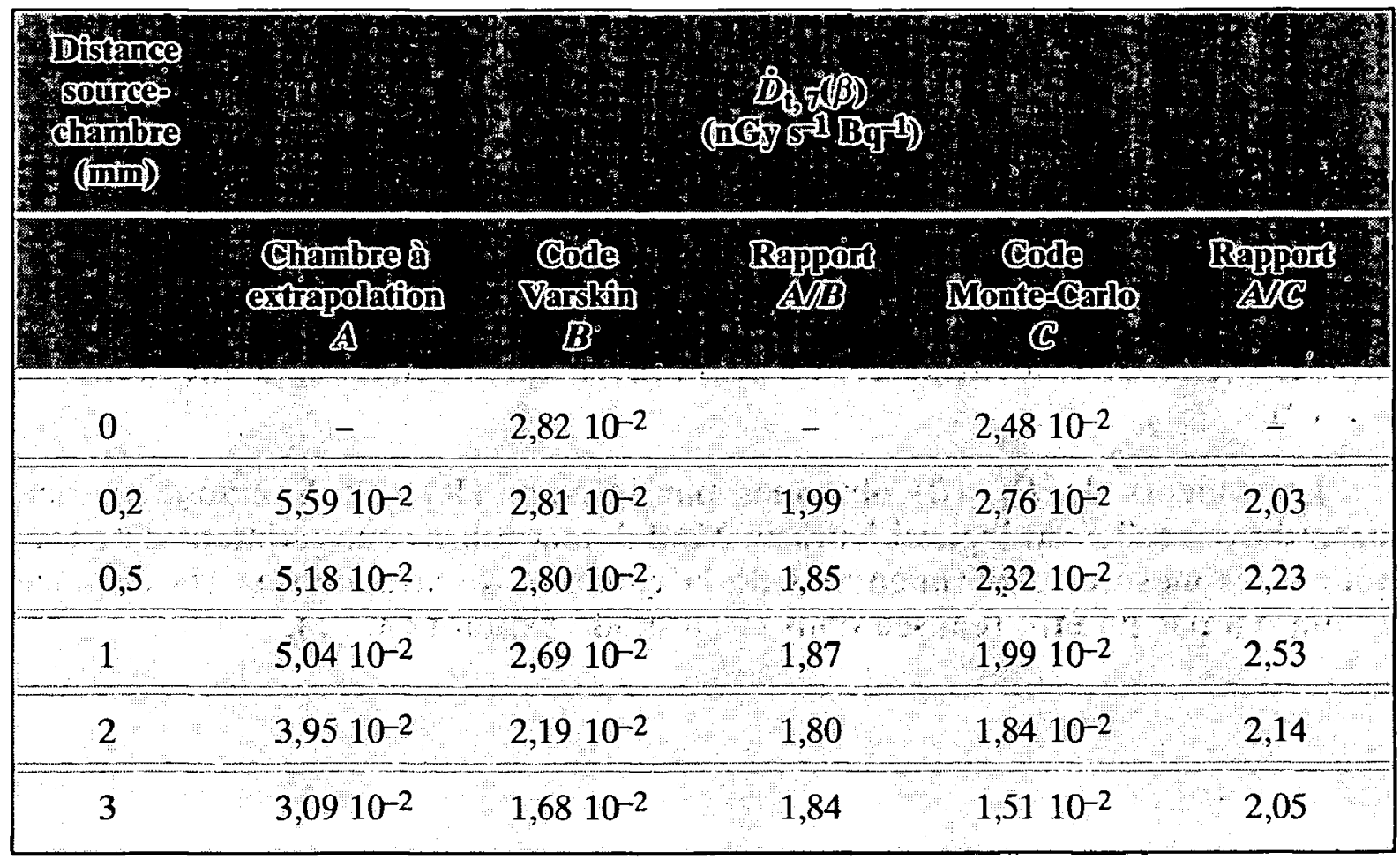


TABLEAU IV

Comparaison des résultats expérimentaux et du code de calcul Varskin Mod 2 pour la dose totale $(\beta+\gamma)$

Comparison of the $(\beta+\gamma)$ total dose rate carried out by experiments and Varskin calculations

\begin{tabular}{|c|c|c|c|}
\hline $\begin{array}{l}\text { Distance } \\
\text { source } \\
\text { chambre } \\
\text { (mm) }\end{array}$ & & 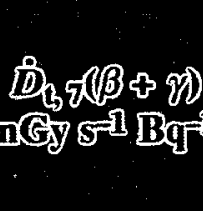 & \\
\hline & $\begin{array}{c}\text { Chambre â } \\
\text { extrapolation } \\
\text { A }\end{array}$ & $\begin{array}{c}\text { Code } \\
\text { Varskin } \\
\text { B }\end{array}$ & $\begin{array}{l}\text { Rapport } \\
A \text { /B }\end{array}$ \\
\hline 0 & - & $4,1810^{-2}$ & - \\
\hline 0,2 & $6,0910^{-2}$ & $4,0610^{-2}$ & 1,50 \\
\hline 0,5 & $5,6810^{-2}$ & $3,9510^{-2}$ & 1,44 \\
\hline 1 & $5,5410^{-2}$ & $3,6310^{-3}$ & 1,53 \\
\hline 2 & $4,3910^{-2}$ & $2,8110^{-3}$ & 1,56 \\
\hline 3 & $3,4410^{-2}$ & $2,1110^{-3}$ & 1,63 \\
\hline
\end{tabular}

$10^{-3} \mathrm{~cm}^{2}$ à $1 \mathrm{~cm}^{2}$, sont représentés en figure 8 . Il faut noter que, au contact, pour la plus petite surface $\left(10^{-3} \mathrm{~cm}^{2}\right), \dot{D}_{\mathrm{t}, 7}$ est égal à $11 \mathrm{nGy} \mathrm{s}^{-1} \mathrm{~Bq}^{-1}$, soit un débit de dose bêta voisin de $29 \mathrm{~Gy} \mathrm{~h}^{-1}$ pour une activité de $7,410^{5} \mathrm{~Bq}(20 \mu \mathrm{Ci})$.

\section{b) Code Monte-Carlo "HOT $25 \mathrm{~S} 2$ "}

Les résultats présentés dans. le tableau III et la figure 6, sont relatifs à des distances source-détecteur comprises entre 0 et $3 \mathrm{~mm}$. Les calculs ont été effectués pour les données suivantes : nombre d'électrons émis : 100000 ; surface irradiée (aire de l'électrode collectrice) : $0,899 \mathrm{~cm}^{2}$; épaisseur d'une tranche : $2,5 \mu \mathrm{m}$.

Les valeurs de $\dot{D}_{\mathrm{t}, 7}(\beta)$ obtenues par le code HOT $25 \mathrm{~S} 2$ sont en bon accord avec celles du logiciel Varksin Mod 2, mais elles sous-estiment d'un facteur 2 les mesures expérimentales de la chambre à extrapolation (la distance $0 \mathrm{~mm}$ n'a pas pu être réalisée dans la méthode expérimentale). 


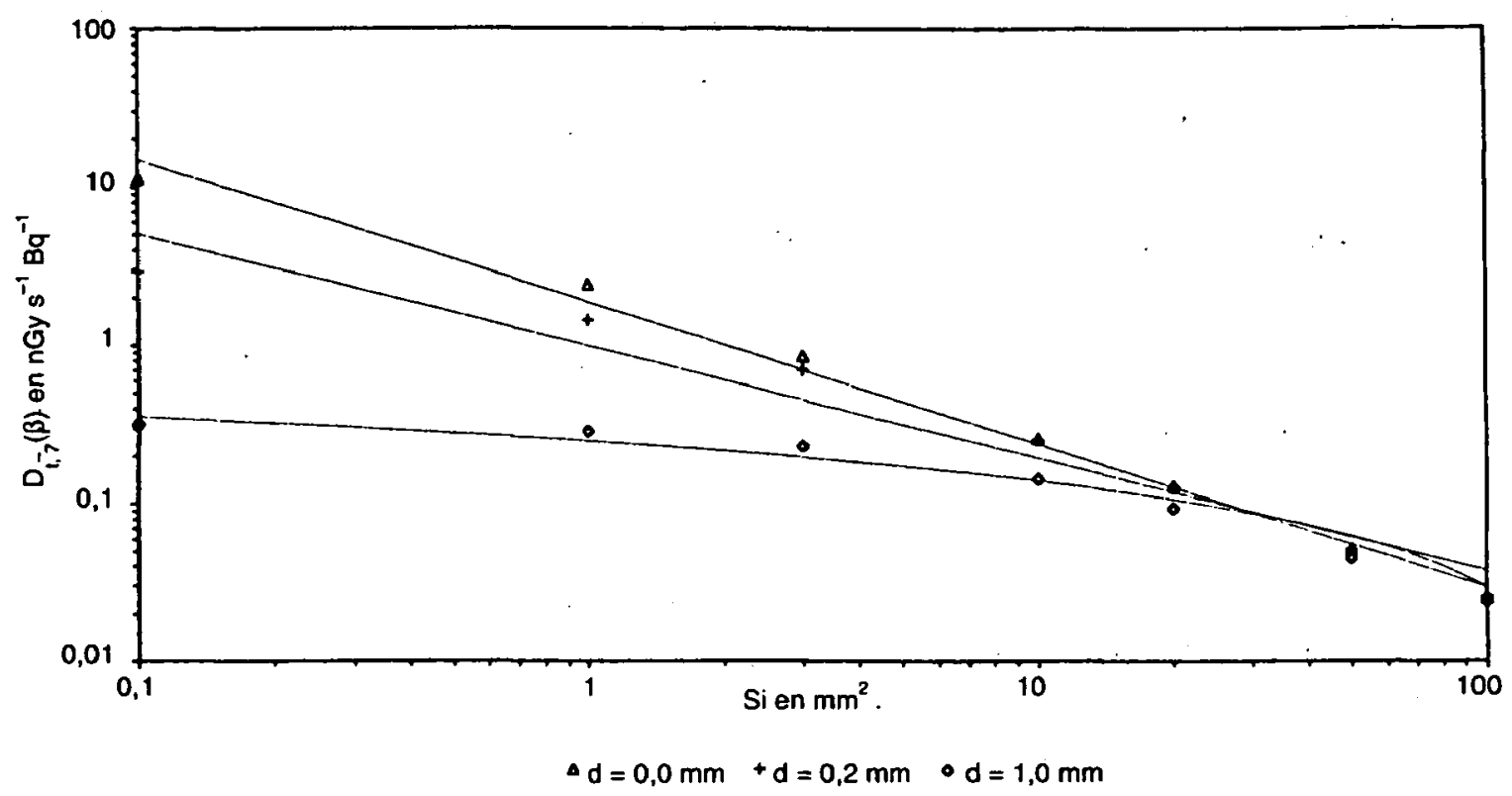

Fig. 8. - Influence de la surface irradiée $S_{\mathrm{i}}$ sur le débit de dose $\beta$ dans le tissu. Irradiated area $S_{\mathrm{i}}$ influence on the $\beta$ tissue dose rate.

\section{Conclusion}

La détermination expérimentale de la dose absorbée bêta, gamma et totale $(\beta+\gamma)$, dans les tissus sous une épaisseur de $70 \mu \mathrm{m}\left(7 \mathrm{mg} \mathrm{cm}{ }^{-2}\right)$, due à une source radioactive de ${ }^{60} \mathrm{Co}$ de très petite dimension (sphère de $200 \mu \mathrm{m}$ de diamètre) a été réalisée au moyen d'une chambre à extrapolation type PTW 23391 équipée d'une électrode collectrice de surface $0,9 \mathrm{~cm}^{2}$, et ceci pour des distances source-détecteur comprises entre $0,2 \mathrm{~mm}$ et $3 \mathrm{~mm}$. Cette source simule le cas d'une particule métallique qui aurait été activée dans un réacteur ("hot particle").

Ces mesures ont été comparées aux valeurs calculées au moyen de deux codes de calcul : le code Varskin Mod 2, écrit par Durham, et le code MonteCarlo HOT 25 S 2 de Patau.

De cette étude, destinée à valider des méthodes de mesure et de calcul pour la dosimétrie bêta au contact de petites sources, il se dégage les conclusions suivantes:

1) La dose totale $(\beta+\gamma)$ calculée par le code Varskin est $50 \%$ plus faible que celle mesurée au moyen de la chambre à extrapolation, bien que la dose gamma calculée soit deux fois plus élevée, mais celle-ci ne représente que $10 \%$ de la dose totale.

2) La dose bêta calculée par.le code Monte-Carlo est en accord avec celle calculée par le code Varskin, mais est deux fois plus faible que la valeur expérimentale. 
En prenant comme base les valeurs "chambre à extrapolation" et "code Varskin", on peut résumer les résultats de la façon suivante, pour une surface irradiée de $1 \mathrm{~cm}^{2}$ :

\begin{tabular}{|lcc|}
\hline Distance source-peau & $0,2 \mathrm{~mm}$ & $1 \mathrm{~mm}$ \\
\hline$\dot{D}_{\mathrm{t}, 7}(\beta+\gamma)$ & $6,110^{-2} \mathrm{nGy} \mathrm{s}^{-1} \mathrm{~Bq}^{-1}$ & $5,510^{-2} \mathrm{nGy} \mathrm{s}^{-1} \mathrm{~Bq}^{-1}$ \\
\hline Dose $\beta$ /Dose $(\beta+\gamma)$ & $90 \%$ & $90 \%$ \\
\hline
\end{tabular}

Il apparaît, d'après les résultats calculés au moyen du code Varskin, que l'évolution du débit de dose bêta entre $0,2 \mathrm{~mm}$ et le contact avec la peau est relativement faible; en conséquence, on peut dire qu'une source sphérique de $60 \mathrm{Co}$ d'activité $7,410^{5} \mathrm{~Bq}(20 \mu \mathrm{Ci})$ et de diamètre $200 \mu \mathrm{m}$ délivre au contact de la peau les débits de dose $(\beta+\gamma)$ suivants :

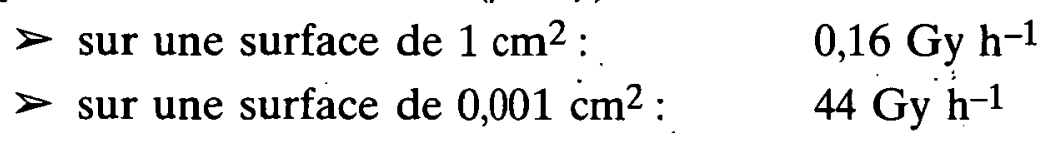

Ceci montre bien l'importance de la surface irradiée, et la nécessité de faire des mesures au moyen d'une chambre à extrapolation équipée d'une électrode de surface plus petite. Ceci a conduit le laboratoire à faire réaliser'par la firme PTW des électrodes de diamètres 2 et $5 \mathrm{~mm}$ (surfaces 3 et $20 \mathrm{~mm}^{2}$ ). Elles seront utilisées pour les prochaines mesures prévues sur des sources de ${ }^{170} \mathrm{Tm}$, dans le cadre d'un nouveau contrat de collaboration avec Charles qui commencera en 1996.

\section{Remerciements}

Ces travaux ont été réalisés dans le cadre des contrats de la Commission des Communautés Européennes B117-021, BI-028 et de la Fiche-Action 4000 du Commissariat à l'Energie Atomique.

\section{RÉFÉRENCES}

Baum J., Kaurin D.G. (1991) Reassessment of data used in setting exposure limits for hot particles. Radiat. Prot. Dosim. 39 (1-3) 49-54.

Berger M.j. (1973) Improved Point Kernels for Electrons and Beta ray Dosimetry. NBSIR 73-107.

Böhm J. (1986) The National Primary Standard of the PTB for Realizing the Unit of the Absorbed Dose Rate to Tissue for Beta Radiation, PTB.DOS.13.

Charles M.W: (1991) The hot particle problem. Radiat. Prot. Dosim. 39 (1-3) 39-47.

Charles M.W., Darley P.J. (1994) Status report on CEC hot particles dosimetry project. F 13-CT 920032/8, March 1994. 
Chibani O. Patau J.P. (1994) New electron multiple scattering distribution for Monte-Carlo transport simulation. Nucl. Instrum. Meth. B 94, 1-10.

Cross W.G. (1986) Variation of Beta Dose Attenuation in Different Media. Phys. Med. Biol. 13 (4) 611-618.

Cross W.G., Ing H., Fredman N. (1989) A short Atlas of Beta Ray Spectra. Phys. Med. Biol. 28 (11) 1251-1260.

Darley P.J., Charles M.W., Hart C.D., Wells J., Coleby M.S.E. (1991) Dosimetry of planar and punctiform beta sources using an automated extrapolation chamber and radiochromic dye films. Radiat. Prot. Dosim. 39 (1-3) 61-66.

Durham J.S., Reece W.D., Merwin S.E. (1991) Modelling three dimensional beta sources for skin dose calculations using Varskin Mod 2. Radiat. Prot. Dosim. 37 (2) 89-94.

Durham J.S. (1991) Hot particle dose calculations using the computer code Varskin Mod 2. Radiat. Prot. Dosim. 39 (1-3) 75-78.

Durham J.S. (1992) Varskin Mod 2 and Sadde Mod 2 ; computer codes for assessing skin dose from skin contamination. NUREG/CR 5873.

Électricité de France (1994). Retour d'expérience parc points chauds.

Hubbell J.H. (1982) Photon Mass Attenuation and Energy absorption Coefficients from $1 \mathrm{keV}$ to $20 \mathrm{MeV}$. Int. J. Radiat. Isot. 33, 1269-1290.

International Commission on Radiological Protection (ICRP) (1991) The Biological Basis for Dose Limitation in the Skin. ICRP. Publication 59.

ICRU (1984) Stopping Powers for Electrons and Positrons. Report 37.

Lantz M.W. and Lambert M.W. (1990) Charged Particle Equilibrum Corrections for the Gamma Component of Hot Particle Skin Doses. Radiat. Prot. Manage. 7 (50) 38-48.

Leroux J.B., Herbaut Y. (1994) Mesures et calculs des doses délivrées par des particules radioactives. Note d'Etude SPR/SMI ${ }^{\circ}$ 94-04.

National Council on Radiation Protection and Measurements (NCRP) (1989) Limit for exposure to "Hot Particles" on the skin. NCRP Report No. 106.

Sociedad Nuclear Espanola (1994) Particulas calientes.

Patau J.P. (1991) Monte-Carlo calculations of the depth dose distribution in skin contaminated by hot particles. Radiat. Prot. Dosim. 39 (1-3) 71-74.

Warnock R.V., Bray L.G., Cooper T.L., Goldin E.M., Knapp P.J., Lewis M.M. and Rigby W.F. (1987) A health physics program for operation with failed fuel. Radiat. Prot. Manage. 4, 21.

Williams M.C., Scannell F.F., Soares M.J., Coursey B.M., Chabotll G.E. (1992) Hot particle dosimetry using ${ }^{60}$ Co spheres. Radiat. Prot. Dosim. 40 (4) 223-234. 